\title{
Primary adenocarcinoma of the appendix in a child: A case report
}

\author{
Toshiaki Takahashi", Hiroshi Nouso, Masaya Yamoto, Koji Fukumoto and Naoto Urushihara
}

\begin{abstract}
Background: Primary adenocarcinoma of the appendix is a rare disease in clinical practice. Moreover, primary adenocarcinoma of the appendix in the pediatric age group is even rarer with very little cases being published. Here, we report a case of primary adenocarcinoma of the appendix with local invasion into adjacent organs in a child who was initially diagnosed as having an acute appendicitis.

Case presentation: A 13-year-old girl presented with abdominal pain of 3-month duration. Imaging study showed a mass including the fecalith that occupied her pelvic and right lower abdominal cavity. Drainage of the abscess and appendectomy were performed by the preoperative diagnosis of an acute appendicitis with an appendiceal mass. Postoperative histopathological examinations revealed the appendiceal adenocarcinoma. She then received the whole mass resection, ileocecal resection with lymph node dissection. The masses were tightly adherent with infiltration into the sigmoid colon, uterus, and right ovary. These organs were all dissected, and subsequent sigmoid colostomy was performed. We preserved the left ovary for her fertility. The pathological findings demonstrated negative margins and no lymph node invasions, and final pathological stage was pT4(SI)NOMO, stage II. After the operation, she received the chemotherapy with 6 cycles of 5-fluorouracil (5-FU), leucovorin (LV), and oxaliplatin (mFOLFOX6) and subsequent 6 cycles of simplified LV and 5-FU (sLV5FU2). The patient is doing well till today on follow-up without progression of the disease 5 years after the operation.

Conclusion: Primary adenocarcinoma of the appendix is exceedingly rare in children. In this report, we described one of the youngest primary adenocarcinomas of an appendix case ever reported. When encountering atypical cases of the appendicitis, we should consider the possibility of primary adenocarcinoma of the appendix as it has an extremely poor prognosis and is usually diagnosed in advanced stages.
\end{abstract}

Keywords: Primary adenocarcinoma, Appendix, Children

\section{Background}

Appendiceal cancer is a rare malignancy with an incidence of around 0.1 in 1,000,000, making up only $0.5 \%$ of all gastrointestinal malignancies [1]. The tumor itself is not aggressive but has the potential for rupture and spread throughout the peritoneum, which carries a poor prognosis [2]. This cancer is most often diagnosed following appendectomy for suspected acute appendicitis [3].

In the pediatric aged group, the incidence of colorectal cancer is rare compared with that in adults. Although early diagnosis and improved management have resulted in reduced mortality since 1980s [4], the treatment

\footnotetext{
* Correspondence: totakaha@juntendo.ac.jp

Department of Pediatric Surgery, Shizuoka Children's Hospital, 860 Urushiyama, Aoi-ku, Shizuoka City, Shizuoka Prefecture 420-8660, Japan
}

protocol of colorectal cancer in children is still controversial due to very little cases being published.

Here, we report a case of primary adenocarcinoma of the appendix (PAA) with local invasion into adjacent organs in a child who was initially diagnosed as having an acute appendicitis.

\section{Case presentation \\ A patient}

A 13-year-old girl presented with abdominal pain and bloody stool. For 3 months, she had repeated appearance of the abdominal pain and spontaneous disappearance of the symptom. At the beginning, the symptom was not strong and she had not seen a doctor. Her abdominal pain gradually got worse, and she determined to come to our hospital after she often had bloody stool. Imaging 
study showed a mass including the fecalith that occupied her pelvic and right lower abdominal cavity (Fig. 1). Drainage of the abscess and appendectomy were performed by the preoperative diagnosis of an acute appendicitis with an appendiceal mass (Fig. 2). Although the body of the appendix looked intact as well as the ileum and the cecum, the histopathological examination revealed PAA in the tip of the resected appendix. A radical operation for the residual tumor of PAA in the pelvic cavity was planned.

\section{Surgery}

She then received the whole mass resection, ileocecal resection with lymph node dissection. At the first operation, we speculate that we resected body of the appendix but left the most of the mass and the tip of the appendix. Therefore, the mass which we resected in the second operation was derived from the tip of the appendix. The masses were tightly adherent with infiltration into the sigmoid colon, uterus, and right ovary. These organs were all dissected, and subsequent sigmoid colostomy was performed (Fig. 3). We preserved the left ovary for her fertility. The pathological findings demonstrated negative margins and no lymph node invasions, and

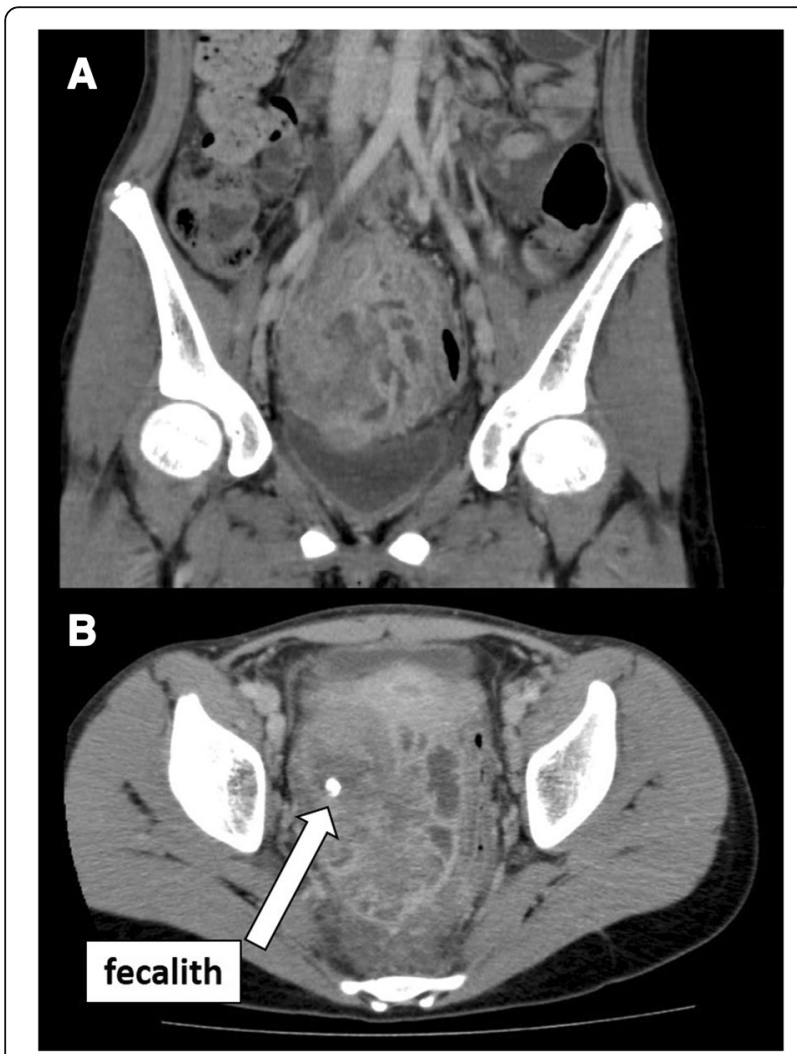

Fig. 1 Imaging study showed a mass including the fecalith that occupied her pelvic and right lower abdominal cavity (a coronal, b horizontal)

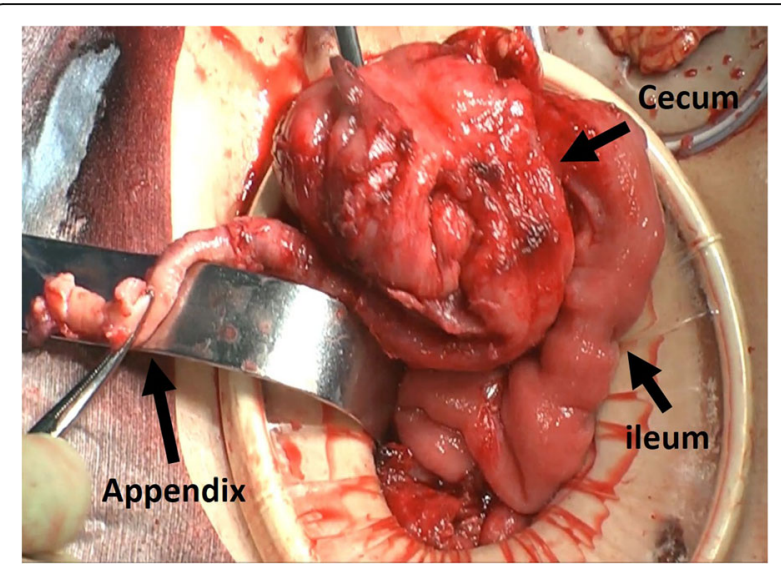

Fig. 2 Intraoperative finding: Drainage of the abscess and appendectomy were performed by the preoperative diagnosis of an acute appendicitis with an appendiceal mass. Although the body of the appendix looked intact as well as the ileum and the cecum, the histopathological examination revealed PAA in the tip of the resected appendix

final pathological stage was pT4(SI)N0M0, stage II (TNM-7th edition 2009). Postoperative course was uneventful.

\section{Postoperative treatment}

After the operation, she received the chemotherapy with 6 cycles of 5-fluorouracil (5-FU), leucovorin (LV), and oxaliplatin (mFOLFOX6) and subsequent 6 cycles of simplified LV and 5-FU (sLV5FU2). The patient is doing well till today on follow-up without progression of the disease 5 years after the operation. We performed the stoma closure operation 2 years after the tumor resection.

\section{Discussion}

Primary adenocarcinoma of the appendix (PAA) is an extremely rare disease in clinical practice [4]. There are

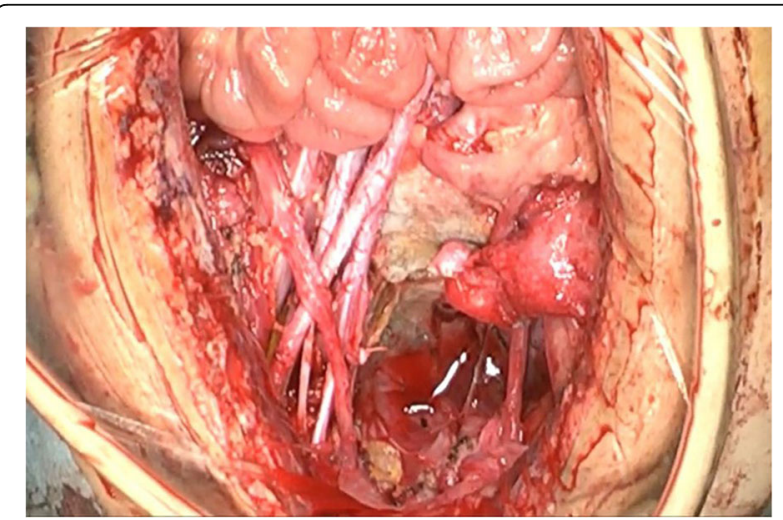

Fig. 3 Intraoperative finding: The patient received the whole mass resection, ileocecal resection with lymph node dissection, and subsequent sigmoidectomy and adnexectomy 
Table 1 The reports of primary colorectal carcinoma in children since 2000

\begin{tabular}{|c|c|c|c|c|c|c|c|}
\hline Case & $\begin{array}{l}\text { Age } \\
\text { (years) }\end{array}$ & Gender & Origin & Pathological results & Operation & Additional treatment & Outcome \\
\hline $1[8]$ & 13 & M & A-colon & Well diff. adenocarcinoma & R-hemicolectomy & - & No recurrence \\
\hline $2[9]$ & 12 & $\mathrm{~F}$ & Rectum & Undiff. mucinous adenocarcinoma & - & Radiotherapy & Die \\
\hline $3[10]$ & 13 & Unknown & D-colon & $\begin{array}{l}\text { Adenocarcinoma with multiple } \\
\text { metastasis }\end{array}$ & $\begin{array}{l}\text { L-hemicolectomy and } \\
\text { splenectomys }\end{array}$ & Chemotherapy & Die \\
\hline $4[11]$ & 9 & M & Rectum & Mucinous adenocarcinoma & segmental resection & Chemotherapy & No recurrence \\
\hline $5[11]$ & 12 & $\mathrm{~F}$ & D-colon & Poorly diff. mucinous adenocarcinoma & resection of local tumor & Chemotherapy & No recurrence \\
\hline 6 [12] & 13 & M & A-colon & Poorly diff. mucinous adenocarcinoma & R-hemicolectomy & Chemotherapy & Die \\
\hline $7[12]$ & 15 & M & T-colon & Mucinous adenocarcinoma & lleosigmoidostomy bypass & Chemotherapy & Die \\
\hline
\end{tabular}

$M$ male, $F$ female, A-colon ascending colon, $D$-colon descending colon, $T$-colon transverse colon, diff. differentiated, $R$-hemicolectomy right hemicolectomy, $L$ hemicolectomy left hemicolectomy

no established risk factors for the development of appendix cancer. Malignant appendix tumors most often present with acute appendicitis and are diagnosed incidentally at histologic assessment of the surgical specimen. Appendix cancers may also be asymptomatic and be found incidentally. When symptoms are present, the disease process is often advanced [5].

Colorectal carcinoma in children, although rarely discovered, comprises approximately $1 \%$ of pediatric neoplasm [6]. It is also the most common primary gastrointestinal malignancy in children. However, due to the low awareness of the disease, diagnosis is usually delayed until the disease is in the advanced stage, causing prognosis to be extremely poor compared with that of adults [7].

In addition, there is no consensus for the goal of the operation for PAA in children. Generally, functional preservation operation has often been emphasized in pediatric surgery. Therefore, it is not easy to decide the grade of the extension in the radical operation for colorectal cancer such as PAA in children as there is very little reports being published. Furthermore, we have no guideline about the postoperative treatment protocol such as the resume of the chemotherapy.
We reviewed some recent literature which has been published since 2000 and found seven cases of the colorectal adenocarcinoma in children who is under 15 years old (Table 1). The tumors originate from the ascending colon in two cases, transverse colon in one case, descending colon in two cases, and rectum in two cases. Most of the cases received adjuvant chemotherapy. Although three cases had good postoperative courses, the patients in four cases died after the radical operation. The poor prognosis of the primary colorectal carcinoma in children has been re-recognized by this review [8-12].

Furthermore, Table 2 shows the reports of primary adenocarcinoma of the appendix in the recent 10 years [13-20]. The mean age at presentation for PAA is about 50 years. There is no sex predominance. Most PAAs are well differentiated, are slowly growing, and have pushing rather than infiltrating margin. The traditional treatment is right hemicolectomy. However, in the case of intra-abdominal metastasis, the treatment consists of aggressive debulking followed by chemo-radiotherapy along with it.

In this report, we demonstrate a case of PAA with local invasion into adjacent organs in a child who was initially diagnosed as having an acute appendicitis. We

Table 2 The reports of primary adenocarcinoma of the appendix in recent 10 years

\begin{tabular}{|c|c|c|c|c|c|c|}
\hline Case & Age (years) & Gender & Pathological results & Operation & $\begin{array}{l}\text { Additional } \\
\text { treatment }\end{array}$ & Outcome \\
\hline $1[13]$ & 49 & M & Well diff. mucinous adenocarcinoma & Tumor resection & Chemotherapy & No recurrence for 15 months \\
\hline $2[14]$ & 57 & M & Mucinous adenocarcinoma & R-hemicolectomy & Chemotherapy & Not mentioned \\
\hline $3[15]$ & 75 & $\mathrm{~F}$ & $\begin{array}{l}\text { Adenocarcinoma arising focally within } \\
\text { an appendiceal tubulovillous adenoma }\end{array}$ & $\begin{array}{l}\text { Resection of the sigmoid colon, } \\
\text { proximal rectum, and appendix }\end{array}$ & Not mentioned & Not mentioned \\
\hline $4[16]$ & 63 & $\mathrm{~F}$ & Mucinous adenocarcinoma & Ileocecal resection & - & No recurrence for 5 years \\
\hline $5[17]$ & 42 & M & $\begin{array}{l}\text { Low-moderate diff. mucinous } \\
\text { adenocarcinoma }\end{array}$ & $\begin{array}{l}\text { Resection of local tumor and } \\
\text { partial bladder }\end{array}$ & Chemotherapy & No recurrence for 6 months \\
\hline $6[18]$ & 35 & $\mathrm{~F}$ & Well diff.mucinous adenocarcinoma & R-hemicolectomy & Chemotherapy & No recurrence \\
\hline $7[19]$ & 80 & M & Signet ring cell adenocarcinoma & R-hemicolectomy & - & No recurrence \\
\hline $8[20]$ & 40 & $\mathrm{~F}$ & Mucinous adenocarcinoma & R-hemicolectomy & - & Not mentioned \\
\hline
\end{tabular}

$M$ male, $F$ female, diff. differentiated, $R$-hemicolectomy right hemicolectomy 
performed radical operation and adjuvant chemotherapy. The patient is doing well without progression of the disease after 5 years. Although little has been understood about PAA, we believe our experience may provide new insights into the guideline of the standard treatment for PAA in children.

\section{Conclusions}

Primary adenocarcinoma of the appendix is exceedingly rare in children. In this report, we described one of the youngest primary adenocarcinoma of appendix case ever reported. When encountering atypical cases of the appendicitis, we should consider the possibility of primary adenocarcinoma of the appendix as it has an extremely poor prognosis and is usually diagnosed in advanced stages.

\section{Abbreviations}

5-FU: 5-Fluorouracil; LV: Leucovorin; PAA: Primary adenocarcinoma of the appendix

\section{Authors' contributions}

TT acquired the data and drafted the manuscript. HN, GM, KO, and NU performed the operations. All other authors attended the patient postoperatively. All authors read and approved the final manuscript.

\section{Ethics approval and consent to participate}

The publication of the present study was undertaken in accordance with the ethical standards of our institution (2018020).

\section{Consent for publication}

Informed consent was obtained from the patient and his family for the publication of this report.

\section{Competing interests}

The authors declare that they have no competing interests.

\section{Publisher's Note}

Springer Nature remains neutral with regard to jurisdictional claims in published maps and institutional affiliations.

Received: 12 June 2018 Accepted: 22 August 2018

Published online: 04 September 2018

\section{References}

1. Murphy EM, Farquharson SM, Moran BJ. Management of an unexpected appendiceal neoplasm. Br J Surg. 2006;93:83-92.

2. Marmor S, Portschy PR, Tuttle TM, Virnig BA. The rise in appendiceal cancer incidence: 2000-2009. J Gaastrointest Surg. 2015;19:743-92750.

3. Misdraji J. Mucinous epithelial neoplasms of the appendix and pseudomyxoma peritonei. Mod Pathol. 2015;28:S67-79.

4. Torre LA, Bray F, Siegel RL, Ferlay J, Lortet-Tieulent J, Jemal A. Grobal cancer statistics. CA Cancer J Clin. 2015;65:87-108.

5. Hamilton W, Round A, Sharp D, Peters TJ. Clinical features of colorectal cancer before diagnosis: a population-based case-control study. Br J Cancer. 2005;93:399-405.

6. Salas-Valverde S, Lizano A, Gamboa Y, Vega S, Barrantes M, Santamaria S, Zamora JB. Colon carcinoma in children and adolescents: prognostic factors and outcome - a review of 11 cases. Pediatr Surg Int. 2009;25:1073-6.

7. Hill DA, Furman WL, Billups CA, Riedley SE, Cain AM, Rao BN, Pratt CB, Squnt SL. Colorectal carcinoma in childhood and adolescence: a clinicopathologic review. J Clin Oncol. 2007;25:5808-14.

8. Ahn CH, Kim SC. Two case reports: colorectal adenocarcinoma in children. Clin Case Rep. 2017;96(46):e8074.

9. Tiwari C, Zadpe A, Rathi P, Shah H. An unusual presentation of rectal carcinoma in a child. Pediatr Gastroenterol Hepathol Nutr. 2018;21:72-5.
10. Singer $G$, Hoellwarth ME. Colorectal carcinomas in children: an institutional experience. Pediatr Surg Int. 2012;28:591-5.

11. Zamir N, Ahmed S, Akhtar J. Mucinous adenocarcinoma of colon. APSP J Case Rep. 2010;1:20.

12. Shih HH, Lu CC, Tiao MM, Ko SF, Chuang JH. Adenocarcinoma of the colon in children presenting as abdominal pain: report of two cases. Chang Gung Med J. 2002;25:349-6354.

13. Dang SC, Sun MM, Liu LL, et al. Giant mucinous adenocarcinoma of the appendix: a case report. J Med Case Rep. 2017;11:206.

14. Handler $\mathrm{M}$, Anand $\mathrm{N}$, Wei $\mathrm{L}$, et al. Adenocarcinoma of the appendix presenting as a palpable right thigh mass. Radiol Case. 2017;11:20-9.

15. Fitzgerald $E$, Chen $L$, Guelrud M, et al. Appendical adenocarcinoma presenting as a rectal polyp. Case Rep Gastroenterol. 2016;10:24-9.

16. Nagata $\mathrm{H}$, Kondo $\mathrm{Y}$, Kawai $\mathrm{K}$, et al. A giant mucinous cystadenocarcinoma of the appendix: a case report and review of the literature. W J Surg Oncol. 2016;14:64

17. Qu R, Jiang Y, Chen S, Dong Q. Primary appendical adenocarcinoma masquerading as primary bladder tumor: a case report and review of literatures. Indian J Surg. 2015;77:S16-8.

18. Koizumi N, Murayama Y, Kuriu Y, et al. Incidentally discovered adenocarcinoma in situ of the appendix in a young woman. Case Rep Gastroenterol. 2012;6:726-33.

19. Fusari M, Sorrentino N, Bottazzi EC, et al. Primary signet ring cell carcinoma of the appendix mimicking acute appendicitis. Case Rep. 2012;1:29.

20. Behera PK, Rath PK, Panda R, et al. Primary appendiceal mucinous adenocarcinoma. Indian J Surg. 2011;73:146-8.

\section{Submit your manuscript to a SpringerOpen ${ }^{\circ}$ journal and benefit from:}

- Convenient online submission

- Rigorous peer review

- Open access: articles freely available online

High visibility within the field

- Retaining the copyright to your article

Submit your next manuscript at $\boldsymbol{\nabla}$ springeropen.com 